\title{
Prenatal Diagnosis of Fetal Hypertrophic Cardiomyopathy in Diabetic Mothers Using 5D Fetal Echocardiography
}

\author{
Mohammad AbdElhameed M. NasrAdDeen, Mohammed Salah Elsokkary, Mohamed Esmat Abbass \\ Shawky, Heba Ramadan Elsayed Abdel Fattah
}

Obstetrics and Gynaecology Department, Faculty of Medicine, Ain Shams University

\begin{abstract}
Background: Diabetes mellitus is a common condition, affecting approximately $7 \%$ of pregnant women. Appropriate management has reduced morbidity and mortality. Diabetes mellitus in pregnancy can cause many problems for the fetus as significant congenital disorders, risk of premature delivery and increased prenatal morbidity and mortality. In addition, it causes neonatal hypoglycemia, macrosomia and transient hypertrophic cardiomyopathy HCM (observed in up to $35 \%$ of fetuses). Fetal Intelligent Navigation Echocardiography (FINE) is a novel method for visualization of standard fetal echocardiography views from volume datasets obtained with spatiotemporal image correlation (STIC).This method can simplify examination of the fetal heart and reduce operator dependency.

Objectives: The aim of this study was to investigate the utility of 5D fetal echocardiography in the prenatal diagnosis of fetal hypertrophic cardiomyopathy in healthy mothers, controlled diabetic mothers and uncontrolled diabetic mothers.

Patients and Methods: Cross sectional observational study was conducted at Ain Shams University Maternity Hospital at the ultrasound fetal special care unit, Study duration: September 2017- July 2018. This study was conducted on healthy and diabetic pregnant women divided into 3 groups Sampling Method: This study included 111 pregnant women between the 28th and 36th weeks of gestation. After approval of the Local Institutional Review Board, the study was explained and an informed written consent was obtained from all participants. This study included 3 groups of pregnant women which were: Group A: Control group which consist of 37 healthy non-diabetic pregnant women, group B: Controlled group which consist of 37 diabetic pregnant women with good glycemic control, group C: Uncontrolled group which consist of 37 diabetic pregnant women with poor glycemic control.

Results: The current study showed that, as regards the thickness of the interventricular septum IVS, there were significant differences between the three groups. The mean IVS thickness in Group A was $0.38 \pm 0.05 \mathrm{~cm}$; in Group B, it was $0.44 \pm 0.05 \mathrm{~cm}$ and in Group C, it was $0.57 \pm 0.08 \mathrm{~cm}$. In a similar study, compared the IVS thickness between GDM, DM type 2 and DM type 1, showed that the mean fetal IVS thickness was $3.73+0.84$ $\mathrm{mm}$ in the DM type1 group; $4.08+0.75 \mathrm{~mm}$ in the GDM group; and $3.99+0.59 \mathrm{~mm}$ in the DM type 2 group and found that there was a statistically significant difference between HbAlc and IVS thickness in the DM type1 group only $\mathrm{P}=0.013$. But in the $\mathrm{GDM}$ group $(\mathrm{P}=0.723)$ and the $\mathrm{DM}$ type 2 group $(\mathrm{P}=0.380)$, there were no significant differences.

Conclusion: Infants of diabetic mothers are prone to have multiple problems during the neonatal period which makes them a very high risk infant. These neonates must be screened for any associated congenital anomalies and metabolic abnormalities. Hypertrophic cardiomyopathy of IDM have good prognosis and this cardiomyopathy is reversible in nature but these neonates requires close monitoring and regular follow-up and early intrauterine detection.
\end{abstract}

Keywords: Prenatal Diagnosis, Fetal Hypertrophic Cardiomyopathy, Diabetic Mothers, 5D Fetal Echocardiography.

\section{INTRODUCTION}

Diabetes mellitus is a common condition, affecting approximately $7 \%$ of pregnant women. Appropriate management has reduced morbidity and mortality ${ }^{(1)}$.

Diabetes mellitus in pregnancy can cause many problems for the fetus as significant congenital disorders, risk of premature delivery and increased prenatal morbidity and mortality. In addition, it causes neonatal hypoglycemia, macrosomia and transient hypertrophic cardiomyopathy HCM (observed in up to $35 \%$ of fetuses) ${ }^{(2)}$.
Approximately 3-6\% of infants of diabetic mothers (IDMs) have congenital cardiac malformations. While $40 \%$ of infants of diabetic mothers have hypertrophic cardiomyopathy (HCM) that may or may not be symptomatic. A major finding is hypertrophy of the ventricular and sepal walls of the neonatal heart. In all, $5 \%$ of neonates born to diabetic mothers suffer from congestive heart failure due to left ventricular outflow obstruction. Fortunately, in most cases, cardiac hypertrophy is transient with spontaneous echocardiographic resolution within the early months after birth, requiring no therapy ${ }^{(3)}$. 
Diabetes mellitus affects the fetal heart during early and late gestation. During early gestation, it hinders the proper expression of genes needed for the correct development of the fetal heart during embryogenesis, causing structural cardiac defects, for example, ventricular septal defects (4). Moreover, during late gestation, fetal hyperinsulinemia due to inadequate maternal glycemic control increases the expression of fetal insulin cardiac receptors. Insulin, an anabolic hormone, causes hyperplasia and hypertrophy of the fetal myocardium specialy the interventricular septum (IVS) due to its abundance of insulin receptors, leading to hypertrophic cardiomyopathy (HCM) ${ }^{(5)}$.

Echocardiography is routinely indicated for foetuses of diabetic women. However, metabolic expression occurs fully from the 24th week onwards ${ }^{(1)}$.

In recent decades, with the development of three-dimensional ultrasound (US3D) and the SpatioTemporal Image Correlation technology (STIC), a new form of prenatal cardiac examination has emerged. This technique allowed obtaining cardiac volume and its storage for later reconstruction and analysis of anatomy, presenting the image in the multiplanar and surface mode (rendered), identifying the cardiac chambers, semilunar and atrioventricular valves, and the positioning of vessels and their correlations, and it was also possible to track the cardiac motion by using the cineloop technique ${ }^{(6)}$.

2D ultrasound is basically an axial image, and $3 \mathrm{D}$ ultrasound is a volume, and $4 \mathrm{D}$ ultrasound is a volume with time and the fifth dimension is how do you bring a level of workflow into ultrasound? And it is basically bordering on the sense of automation.5D technology is a form of automation where you go through and do a scan and you get the results auto populated for you. Five dimensional Ultrasound included features like 5D Heart, 5D CNS (which is aimed at the central nervous system and displays six measurements (BPD, HC, OFD, Cerebellum, Posterior Fossa, Atria lateral ventricle)), 5D Follicle (which identifies and measures multiple ovarian follicles for rapid assessment of follicular size), 5DLB (fetal long-bone) and 5D-NT (nuchal translucency) ${ }^{(7)}$.

Fetal Intelligent Navigation Echocardiography (FINE) is a novel method for visualization of standard fetal echocardiography views from volume datasets obtained with spatiotemporal image correlation (STIC).This method can simplify examination of the fetal heart and reduce operator dependency ${ }^{(8)}$.
It is a method to: 1) demonstrate nine cardiac diagnostic planes; and 2) spontaneously navigate the anatomy surrounding each of the nine cardiac diagnostic planes (Virtual Intelligent Sonographer Assistance (VIS-Assistance)) ${ }^{(9)}$.

\section{AIM OF THE WORK}

The aim of this study was to investigate the utility of 5D fetal echocardiography in the prenatal diagnosis of fetal hypertrophic cardiomyopathy in healthy mothers, controlled diabetic mothers and uncontrolled diabetic mothers. Study Question: Does $5 \mathrm{D}$ fetal echocardiography have a role in the prenatal diagnosis of fetal hypertrophic cardiomyopathy in healthy mothers, controlled diabetic mothers and uncontrolled diabetic mothers? Study Hypothesis: We assumed that 5D fetal echocardiography has a role in the prenatal diagnosis of fetal hypertrophic cardiomyopathy in healthy mothers, controlled diabetic mothers and uncontrolled diabetic mothers. Study Outcomes: 1. Primary outcome: measuring of interventricular septum thickness (IVS) of the fetal heart by $5 \mathrm{D}$ ultrasound. 2. Secondary outcome: Measuring of right myocardial wall thickness (RMWT) of the fetal heart by 5D ultrasound, measuring of left myocardial wall thickness (LMWT) of the fetal heart by 5D ultrasound, finding of any other structural abnormalities of the fetal heart by $5 \mathrm{D}$ ultrasound.

\section{PATIENTS AND METHODS}

Type of Study: Cross sectional
observational study.

Study Setting: This study was conducted at Ain Shams University Maternity Hospital at the ultrasound fetal special care unit. The study was approved by the Ethics Board of Ain Shams University and an informed written consent was taken from each participant in the study.

Study duration: September 2017- July 2018.

\section{Study Population:}

This study was conducted on healthy and diabetic pregnant women divided into 3 groups with the following inclusion and exclusion criteria:

Inclusion Criteria: Pregnant women at 28 to 36 weeks of gestation determined by the last menstrual period (LMP) confirmed by ultrasound test performed until the 10th week, using the crown-rump length (CRL) as a parameter. Women with singleton pregnancy with live 
embryo. Fulfil criteria of FINE (Fetal Intelligent Navigation Echocardiography). Fetal spine located between 5 to 7 o'clock positions (reducing the possibility of shadowing from the ribs or spine). Upper mediastinum and stomach contained within the volume.

Exclusion Criteria: Oligohydramnios [amniotic fluid index (AFI) below the 5th percentile for gestational age. Estimated fetal weight below two standard deviations from the average. Pregnant women with chronic illnesses that could affect fetal growth and development, such as hypertension, collagen diseases. Maternal conditions where intense sound beam could be attenuated such as maternal obesity and abdominal scars. Fetal malformations diagnosed at 1st or 2nd trimester ultrasound. Criteria when fetal Echocardiography could not be done. Fetal spine anterior. Multiple acoustic shadows. Smokers.

Sampling Method: This study included 111 pregnant women between the 28th and 36th weeks of gestation. After approval of the Local Institutional Review Board, the study was explained and an informed written consent was obtained from all participants. The diagnosis of diabetes was based on the criteria provided by the American Diabetes Association, that was, plasma glucose level $>92 \mathrm{mg} / \mathrm{dl}$ (fasting) and $>153 \mathrm{mg} / \mathrm{dl}$ ( 2 hours post prandial), (According to American Diabetes Association (ADA) 2016 Guideline): We measured the glycosylated hemoglobin (HbAlc) levels of the participants; the mean cut off value was $6.5 \%$, where a level of $<6.5 \%$ indicates good glycemic control and a level of $>6.5 \%$ indicates poor glycemic control.

This study included 3 groups of pregnant women which are: Group A: Control group which consist of 37 healthy non-diabetic pregnant women. Group B: Controlled group which consist of 37 diabetic pregnant women with good glycemic control. Group C: Uncontrolled group which consist of 37 diabetic pregnant women with poor glycemic control.

Study Procedures: After enrolment, all women were subjected to the following: Complete history taking, physical examination, investigations, ultrasound examination.

\section{Statistical Analysis:}

The required sample size has been calculated using the IBM $\odot$ SamplePower $\odot$ Software (IBM@ Corp., Armonk, NY, USA).

\section{RESULTS}

Table (1): Comparison between the three studied groups according to obstetric data.

\begin{tabular}{|c|c|c|c|c|c|c|c|c|}
\hline \multirow{2}{*}{$\begin{array}{c}\text { Obstetrics } \\
\text { data }\end{array}$} & \multicolumn{2}{|c|}{$\begin{array}{c}\text { Group A } \\
(\mathbf{n}=\mathbf{3 7})\end{array}$} & \multicolumn{2}{|c|}{$\begin{array}{c}\text { Group B } \\
(\mathbf{n}=37)\end{array}$} & \multicolumn{2}{|c|}{$\begin{array}{c}\text { Group C } \\
(\mathbf{n}=37)\end{array}$} & \multirow[t]{2}{*}{ Test of sig } & \multirow[t]{2}{*}{$\mathbf{p}$} \\
\hline & No. & $\%$ & No. & $\%$ & No. & $\%$ & & \\
\hline \multicolumn{9}{|c|}{ No. Of Deliveries } \\
\hline Min. - Max. & \multicolumn{2}{|c|}{$0.0-5.0$} & \multicolumn{2}{|c|}{$1.0-4.0$} & \multicolumn{2}{|c|}{$0.0-5.0$} & \multirow{2}{*}{$\mathrm{H}=0.932$} & \multirow{2}{*}{0.628} \\
\hline Median & \multicolumn{2}{|c|}{2.0} & \multicolumn{2}{|c|}{2.0} & \multicolumn{2}{|c|}{2.0} & & \\
\hline No abortion & 18 & 48.6 & 18 & 48.6 & 3 & 8.1 & \multirow{2}{*}{$c^{2}=17.788^{*}$} & \multirow{2}{*}{$<0.001^{*}$} \\
\hline Abortion & 19 & 51.4 & 19 & 51.4 & 34 & 91.9 & & \\
\hline Min. - Max. & \multicolumn{2}{|c|}{$1.0-3.0$} & \multicolumn{2}{|c|}{$1.0-6.0$} & \multicolumn{2}{|c|}{$1.0-3.0$} & \multirow{2}{*}{$\mathrm{H}=1.400$} & \multirow{2}{*}{0.496} \\
\hline Median & \multicolumn{2}{|c|}{2.0} & \multicolumn{2}{|c|}{2.0} & \multicolumn{2}{|c|}{2.0} & & \\
\hline
\end{tabular}

This table shows that there was no significant difference between the three groups as regards deliveries and abortion but as regards occurrence of previous abortion group $\mathrm{C}$ had higher percentage.

Table (2): Comparison between the two studied groups according to type of DM

\begin{tabular}{|l|c|c|c|c|c|c|}
\hline \multirow{2}{*}{ DM } & \multicolumn{2}{|c|}{$\begin{array}{c}\text { Group B } \\
(\mathbf{n = 3 7})\end{array}$} & \multicolumn{2}{|c|}{$\begin{array}{c}\text { Group C } \\
(\mathbf{n}=\mathbf{3 7})\end{array}$} & \multirow{2}{*}{ Test of sig. } & \multirow{2}{*}{ p } \\
\cline { 2 - 5 } & No. & \% & No. & \% & & \\
\hline Type & & & & & \multirow{2}{*}{$\chi^{2}=$} & \multirow{2}{*}{$<0.001^{*}$} \\
\hline GDM & 32 & 86.5 & 11 & 29.7 & \multirow{2}{*}{$150.387^{*}$} & \\
\hline Type 1 & 2 & 5.4 & 16 & 43.2 & \\
\hline Type 2 & 3 & 8.1 & 10 & 27.0 & & \\
\hline
\end{tabular}

This table indicates that there were significant differences between the two groups as regards type of diabetes as in group B. The commonest type was GDM, group C Type 1 was the commonest type.

Table (3): Comparison between the two studied groups according to $\mathrm{HbA1c}$ level

\begin{tabular}{|c|c|c|c|c|}
\hline HbA1c & $\begin{array}{c}\text { Group B } \\
(\mathbf{n}=37)\end{array}$ & $\begin{array}{c}\text { Group C } \\
(\mathbf{n}=37)\end{array}$ & Test of sig. & $\mathbf{p}$ \\
\hline Min. - Max. & $4.40-6.40$ & $7.0-10.10$ & \multirow{3}{*}{$\mathrm{t}=14.983^{*}$} & \multirow{3}{*}{$<0.001^{*}$} \\
\hline Mean \pm SD. & $5.52 \pm 0.67$ & $8.36 \pm 0.94$ & & \\
\hline Median & 5.60 & 8.20 & & \\
\hline
\end{tabular}

This table demonstrates that there were significant differences between the two groups as regards $\mathrm{HbAlc}$ as it was higher in group $\mathrm{C}$.

Table (4): Comparison between the three studied groups according to IVS

\begin{tabular}{|c|c|c|c|c|c|}
\hline & $\begin{array}{c}\text { Group A } \\
(\mathbf{n}=\mathbf{3 7})\end{array}$ & $\begin{array}{c}\text { Group B } \\
(\mathbf{n}=37)\end{array}$ & $\begin{array}{c}\text { Group C } \\
(\mathbf{n}=37)\end{array}$ & $\mathbf{F}$ & $\mathbf{p}$ \\
\hline \multicolumn{6}{|l|}{ IVS (in cm) } \\
\hline Min. - Max. & $0.31-0.51$ & $0.36 \pm 0.54$ & $0.45-0.72$ & \multirow{3}{*}{$92.425^{*}$} & \multirow{3}{*}{$<0.001^{*}$} \\
\hline Mean \pm SD. & $0.38 \pm 0.05$ & $0.44 \pm 0.05$ & $0.57 \pm 0.08$ & & \\
\hline Median & 0.38 & 0.43 & 0.57 & & \\
\hline Sig. bet. grps & \multicolumn{3}{|c|}{$\mathrm{p}_{1}<0.001^{*}, \mathrm{p}_{2}<0.001^{*}, \mathrm{p}_{3}<0.001^{*}$} & & \\
\hline
\end{tabular}

This table displays that there were significant differences between the three groups as regards IVS. 


\section{DISCUSSION}

This study was conducted at Ain Shams University Maternity Hospital at the ultrasound fetal special care unit over the period from September 2017 to July 2018.The aim of this study was to investigate the utility of 5D fetal echocardiography in the prenatal diagnosis of fetal hypertrophic cardiomyopathy (HCM) in healthy mothers, controlled diabetic mothers and uncontrolled diabetic mothers. 111 pregnant women between the 28th and 36th weeks of gestation were included and divided into three groups: Group A: Control group which consist of 37 healthy non-diabetic pregnant women. Group B: Controlled diabetic group which consist of 37 diabetic pregnant women with good glycemic control. Group $C$ : Uncontrolled diabetic group which consist of 37 diabetic pregnant women with poor glycemic control.

The current study showed that, as regards the thickness of the interventricular septum IVS, there were significant differences between the three groups. The mean IVS thickness in Group A was $0.38 \pm 0.05 \mathrm{~cm}$; in Group B, it was $0.44 \pm 0.05 \mathrm{~cm}$ and in Group C, it was $0.57 \pm 0.08 \mathrm{~cm}$.

In a similar study, comparison was done between the IVS thickness between GDM, DM type 2 and DM type 1 . The results showed that the mean fetal IVS thickness was $3.73 \pm 0.84 \mathrm{~mm}$ in the DM type1 group; $4.08 \pm 0.75 \mathrm{~mm}$ in the GDM group; and $3.99 \pm 0.59 \mathrm{~mm}$ in the DM type 2 group. Moreover, it was found that there was a statistically significant difference between HbAlc and IVS thickness in the DM type 1 group only $\mathrm{P}=0.013$. But in the $\mathrm{GDM}$ group $(\mathrm{P}=0.723)$ and the $\mathrm{DM}$ type 2 group $(\mathrm{P}=0.380)$, there were no significant differences ${ }^{(10)}$.

Kosus et al. indicated that the mean IVS thickness was slightly higher in the second group, which consisted of 31 patients having glucose levels above $130 \mathrm{mg} / \mathrm{dl}$ after a $50 \mathrm{~g}$ OGL (oral glucose loading) but a normal OGTT, from the first group of 44 patients with normal OGL and OGTT, but it was not statistically significant different between the two groups. The author discovered four GDM cases where the mean IVS thickness was $2.72 \pm 0.13 \mathrm{~mm}$. Because the very small sample size, statistical evaluation was not done for the GDM group ${ }^{(11)}$.

The mechanisms by which interventricular septal hypertrophy arises in the presence of wellcontrolled diabetic pregnancies remain to be unclear. On the other hand, in another study assessing ventricular hypertrophy in patients with mild GIGT (gestational impaired glucose tolerance), a slightly thicker IVS was found in these patients, but there was no statistically significant difference when compared to a control group consisting of normal pregnant women ${ }^{(12)}$.

In the present study, we showed that there were significant differences between the three groups as regard right myocardial wall thickness (RMWT) and left myocardial wall thickness (LMWT) as it was higher in group $\mathrm{C}$ p-value $<0.001$. The mean RMWT were $0.42 \pm 0.06 \mathrm{~cm}$, $0.46 \pm 0.05 \mathrm{~cm}$ and $0.59 \pm 0.09 \mathrm{~cm}$, respectively, in group A,B and C. The mean LMWT were $0.47 \pm$ $0.09 \mathrm{~cm}, 0.52 \pm 0.05 \mathrm{~cm}$ and $0.68 \pm 0.06 \mathrm{~cm}$, respectively, in group $\mathrm{A}, \mathrm{B}$ and $\mathrm{C}$.

Similar findings were found in a previous study that used left ventricular wall thickness measures at birth as reference, it was reported that these values were increased in $69 \%$ of the fetuses of mothers with GDM and that was statistically significant different ${ }^{(13)}$.

In this study, there was a significant correlation between IVS vs. RMWT in group A, B, there was no significant correlation between them in group $\mathrm{C}$.There was a significant correlation between IVS vs. LMWT in group A, B but there was no significant correlation between them in group $\mathrm{C}$.There was a significant correlation between RMWT vs. LMWT in the three groups.

Also in the present study, we found that sensitivity and specificity of IVS in diagnosing diabetic HCM among group B,C vs. A was $85.14 \%$, and $81.08 \%$, respectively, with cut off value $>0.41$,PPV 90,NPP,73.2,Sensitivity and specificity of LMWT in diagnosing diabetic HCM was $85.14 \%$, and $78.38 \%$,respectively, with cut off value $>0.5$,PPV 88.7,NPP, 72.5,Sensitivity and specificity of RMWT in diagnosing diabetic HCM was $78.38 \%$, and $72.97 \%$, respectively, with cut off value $>0.43$, PPV 85.3,NPP 62.8.

Sensitivity and specificity of IVS in diagnosing diabetic HCM Group C vs. group B was $91.89 \%$, and $83.78 \%$,respectively, with cut off value >0.56,PPV 85.0,NPP 91.2. Sensitivity and specificity of LMWT in diagnosing diabetic HCM was $89.19 \%$,and $86.49 \%$. Respectively. with cut off value >0.48,PPV 86.8,NPP88.9. Sensitivity and specificity of RMWT in diagnosing diabetic HCM was $91.89 \%$, and $83.78 \%$, respectively, with cut off value $>0.49$,PPV 85.0,NPP 91.2. 
This shows that they had higher sensitivity and specificity and NPV, PPV in diagnosing diabetes $\mathrm{HCM}$ in controlled and non controlled patients.

A prenatal IVS thickness of $\geq 4.5 \mathrm{~mm}$ was associated with a postnatal diagnosis of HCM at a sensitivity of $82 \%$, a specificity of $68 \%$, a positive predictive value (PPV) of $37 \%$, a negative predictive value (NPV) of $94 \%$, a positive likelihood ratio (LR+) of 2.6, and an overall accuracy of $72 \%$.ROC curve, which was constructed for estimating the association between the prenatal IVS thickness to left myocardial thickness (IVS/LMWT) ratio and the postnatal diagnosis of HCM, showed a significant association ${ }^{(5)}$.

In the present study, we found that there were no significant differences between studied groups as regards age, deliveries and gestational age p-value $0.25,0.628$, and 0.140 , respectively, but as regard occurrence of previous abortion group $\mathrm{C}$ had higher percentage, there was significant differences between the two groups $(B+C)$ as regard type of diabetes as in group $\mathrm{B}$ the commonest type was GDM, group C Type 1 was the commonest type.

In our study we concluded that there were significant differences between the two groups $(\mathrm{B}+\mathrm{C})$ as regard HbA1c, FGT and 2hr OGTT as it was higher in group $\mathrm{C}$. The mean HbA1c in Group $B$ was $5.52 \pm 0.67$ and in Group C 8.36 \pm 0.94 . While, the mean FGT in Group A was $74.68 \pm 7.88$, in Group B 88.76 \pm 17.12 and in Group C 146.1 \pm 63.45 .As regards $2 \mathrm{hr}$ OGTT we found that the mean value was in Group A $127 \pm 17.61$, in Group B 131.5 \pm 23.99 and in group C 198.5 \pm 50.47 .

In the study of Babović et al. which aimed to determine $\mathrm{HbA1c}$ levels and pregestational body mass index BMI as the predictors of glycemic control and fetal IVS thickness, it showed a statistically significant relation between BMI and HbA1c in DM type 1 group only not in DM type 2 and GDM groups. Also, it showed a statistically significant relation between BMI and IVS thickness in the DM type 1 group only ${ }^{(10)}$.

Finally, this study was conducted on healthy and diabetic pregnant women and it showed that there were significant differences between HbA1c and IVS, RMWT, LMWT in the three groups. It might be used as a predictor of HCM in fetuses of diabetic mothers and as a predictor of glycemic control status. This study missed pregetational BMI of the participants. Also, for neonatal outcome it is better to be evaluated by post natal echocardiography.

\section{CONCLUSION}

Infants of diabetic mothers are prone to have multiple problems during the neonatal period which makes them very high risk infants. These neonates must be screened for any associated congenital anomalies and metabolic abnormalities. Hypertrophic cardiomyopathy of IDM have good prognosis and this cardiomyopathy is reversible in nature but these neonates requires close monitoring and regular follow-up and early intrauterine detection.

\section{REFERENCES}

1. Zielinsky P, Luchese S, Manica JL, Piccoli AL, Nicoloso LH, Leite MF et al. (2009): Left atrial shortening fraction in fetuses with and without myocardial hypertrophy in diabetic pregnancies. Ultrasound in obstetrics \& gynecology: the official journal of the International Society of Ultrasound in Obstetrics and Gynecology, 33(2): 182-187.

2. Cimen D and Karaaslan S (2014): Evaluation of cardiac functions of infants of diabetic mothers using tissue Doppler echocardiography. Turkish Archives of Pediatrics, 49: 25-29.

3. Leipold H, Worda C, Schwindt J, KautzkyWiller A, Bancher-Todesca D, Husslein PW (2005): Severe diabetic fetopathy despite strict metabolic control. The Central European Journal of Medicine, 117(15): 561-564.

4. Molin DG, Roest PA, Nordstrand H, Wisse LJ, Poelmann RE, Eriksson UJ et al. (2004): Disturbed morphogenesis of cardiac outflow tract and increased rate of aortic arch anomalies in the offspring of diabetic rats. Birth defects research Part A, Clinical and Molecular Teratology, 70(12): 927-938.

5. Elmekkawi SF, Mansour GM, Elsafty MS, Hassanin AS, Laban M, Elsayed HM (2015): Prediction of Fetal Hypertrophic Cardiomyopathy in Diabetic Pregnancies Compared with Postnatal Outcome. Clinical medicine insights Women's health, 8: 39-43. 
6. Rolo LC, Marcondes M, Araujo J, Simioni C, Maccagnano Z, Fernandes $M$ (2011): Reference curve of the fetal ventricular septum area by the STIC method: preliminary study. Brazilian Cardiology Archives, 96(5): 386-392.

7. Liza H (2015): http:// www. Diagnostic imaging. com/ ultrasound/ more-functionality5d-ultrasound.

8. Garcia M, Yeo L, Romero R, Haggerty D, Giardina I, Hassan SS et al. (2016): Prospective evaluation of the fetal heart using Fetal Intelligent Navigation Echocardiography (FINE). Ultrasound in obstetrics \& gynecology: the official journal of the International Society of Ultrasound in Obstetrics and Gynecology (ISUOG), 47(4): 450-459.

9. Yeo L and Romero R (2013): Fetal Intelligent Navigation Echocardiography (FINE): a novel method for rapid, simple, and automatic examination of the fetal heart. Ultrasound in obstetrics \& gynecology: the official journal of the International Society of Ultrasound in Obstetrics and Gynecology (ISOUG), 42(3): 268-284.

10. Babović I, Arandjelović $M$, Plešinac $S$ et al. (2018): Materna 1 glycoregulation in pregnancies complicated by diabetes mellitus in the prediction of fetal echography findings and perinatal outcomes. J. Obstet. Gynaecol. Res., 44(3): 432-439.
11. Kosus A, Kosus N, Turhan NO (2011): Assessment of cardiomyopathy in fetuses of women with false positive oral glucose loading test. European Journal of Obstetrics \& Gynecology and Reproductive Biology, 154: 37-39.

12. Wong ML, Wong WHS, Cheung YF (2007): Fetal myocardial performance in pregnancies complicated by gestational impaired glucose tolerance. Ultrasound Obstet Gynecol., 29:395-400.

13. Han SS, Wang G, Jin Y et al. (2015): Investigating the mechanism of hyperglycemia-induced fetal cardiac hypertrophy. PLoS One, 10(09):e0139141. 